\title{
Coulisses
}

Revue de théâtre

25| Hiver 2002

Varia

\section{Montagne Froide, pour un art en changement}

Entretien avec Valentine Verhaeghe, par Yossi Rosenboim

\section{Yossi Rosenboim}

\section{OpenEdition}

\section{Journals}

Édition électronique

URL : http://journals.openedition.org/coulisses/5915

DOI : 10.4000/coulisses.5915

ISSN : 2546-9460

Éditeur

Presses universitaires de Franche-Comté

\section{Édition imprimée}

Date de publication : 1 janvier 2002

Pagination : 19-23

ISBN : 2-84627-052-X

ISSN : $1150-594 \mathrm{X}$

Référence électronique

Yossi Rosenboim, « Montagne Froide, pour un art en changement », Coulisses [En ligne], 25 | Hiver 2002, mis en ligne le 24 octobre 2019, consulté le 15 novembre 2019. URL : http://

journals.openedition.org/coulisses/5915; DOI : 10.4000/coulisses.5915

Ce document a été généré automatiquement le 15 novembre 2019.

Coulisses 


\title{
Montagne Froide, pour un art en changement
}

\author{
Entretien avec Valentine Verhaeghe, par Yossi Rosenboim
}

Yossi Rosenboim

Yossi Rosenboim. - Quelle inspiration vous a permis de commencer cette aventure culturelle/artistique?

Montagne Froide. Montagne Froide est le nom d'un lieu, emprunté par le poète Han Shan, Mont Froid, en Chine du Sud à l'époque Tang. Han Shan porte un regard amusé et détaché sur le quotidien, il est souvent représenté devisant avec un compagnon, Shi Te, balayeur joyeux et libre en limite du temple, trop libre pour s'enfermer avec les doctes moines. Montagne Froide est un millefeuille ${ }^{1}$ où se rencontrent plusieurs champs de recherche et d'expériences, poésie, arts plastiques, danse, performance, arts électroniques, édition (...) tissés le plus souvent au sein d'actions intermedia, en lien aussi avec un atelier de recherche théorique. Cette production expérimentale trouve une visibilité lors d'expositions, d'actions, d'édition et d'organisation d'événements. Oui, c'est dire l'importance de la rencontre avec l'œuvre de Han Shan, dont les textes traduits dès les années cinquante ont été fondamentaux pour nombre de poètes, notamment pour les écrivains Américains. C'est aussi la rencontre inspirante avec des poètes contemporains, Henri Chopin, Claude Pélieu, Julien Blaine (...), avec l'écrivain Claude Louis-Combet, avec les danseurs Kazuo Ono, Dominique Petit, avec des agitateurs. L'importance de Polyphonix et de Jean-Jacques Lebel est certaine, mais aussi celle de Michel Giroud dispersant la dispersion, réalisant une formidable connexion, notamment au travers de la revue Kanal, de réseaux de réseaux de connexions transmedia, travaillant sans cesse à une œuvre en mouvement. L'importance première d'artistes tel Robert Filli dont l'œuvre polymorphe et complexe est souvent pleine d'humour et de simplicité, Robert Filliou auteur de textes de théâtre ${ }^{2}$, poète, théoricien, je pense à ce beau livre Teaching and learning ${ }^{3}$, Robert Filliou « briquoleur » de génie. 

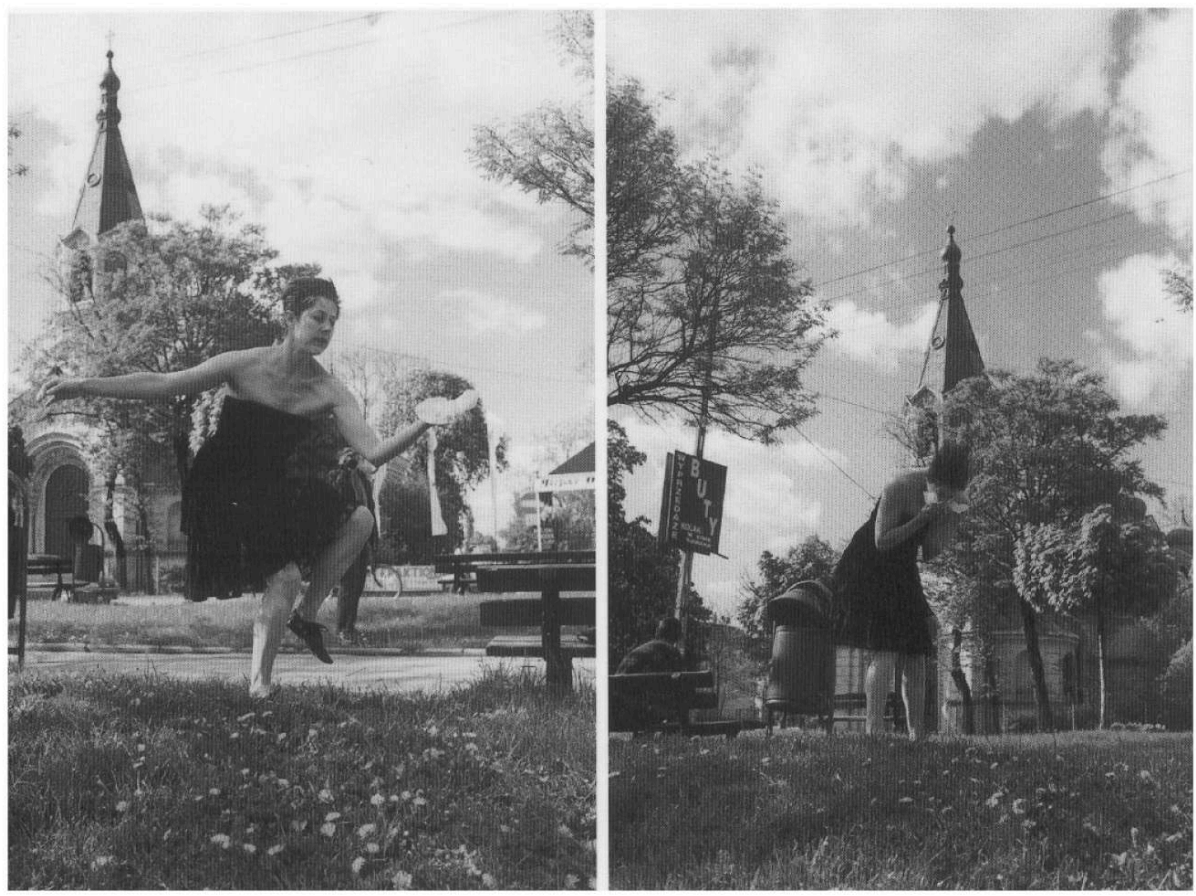

Photo : Patrick Bailly

\section{Y. R. - Y A-T-IL UNE DIMENSION NÉCESSAIRE DANS VOS PERFORMANCES ?}

Montagne Froide n'est pas identifiable à une personne, c'est un groupe de travail, un ensemble aux frontières souples qui en fonction des projets en travail, associe plusieurs chercheurs, artistes, compositeurs, écrivains (...). C'est un organisme vivant. Et l'une des dimensions communes aux différentes actions de Montagne Froide est certainement l'écoute, l'attention au contexte, au paysage et à ses habitants. Pas de volonté de maîtrise. Nous acceptons la possibilité de l'erreur; la faute qui renvoie en fait à l'idée d'écart, écart à la moyenne, métaphore des savants travaux d'Ilya Prigogine qui théorise cet écart dissipateur d'énergie, dissipation permettant qu'advienne un niveau d'élaboration supérieure. Une bifurcation productive de questions et d'évolutions jusque-là imprévisibles.

\section{Y. R. - pour montagne froide, y A-T-IL UNe RÉponse à chaque QUestion, et Vice VERSA?}

Nous apparentons notre activité à la construction d'un panorama, à une mise en scène - dans le sens de skênê, la tente - d'une expérience de pensée, de relations, de création, la scène comme tente et comme indice de mobilité. Montagne Froide ne dispose pas d'un lieu particulier. Le travail se déroule ainsi dans des lieux qui ne sont pas toujours spécialisés pour l'art, par exemple une forêt, une rivière, un marché, un autobus, un café à la campagne (...) mais aussi dans un théâtre, un musée ou une galerie, à Bremen ou à Héricourt, à Barcelone ou à Ventabren, à Périgueux ou Genova (...). Montagne Froide regroupe des "généralistes» suivant l'expression d'Allan Kaprow $^{4}$ et non pas «des spécialistes de l'art », en ce moment Michel Collet anime une recherche portant précisément sur cette idée de Divers. 5 . Pour en revenir à votre question, nous tentons de construire des dispositifs permettant à chacun, acteur et visiteur, de mettre en jeu des processus d'accommodation, de jouer de focales différentes, de déplacer les points de vue. On sait que le changement de point de vue, 
conduit à l'énonciation de nouvelles questions. Si vous voulez, la construction de la scène s'opère dans le mouvement. Et là, nous dansons.

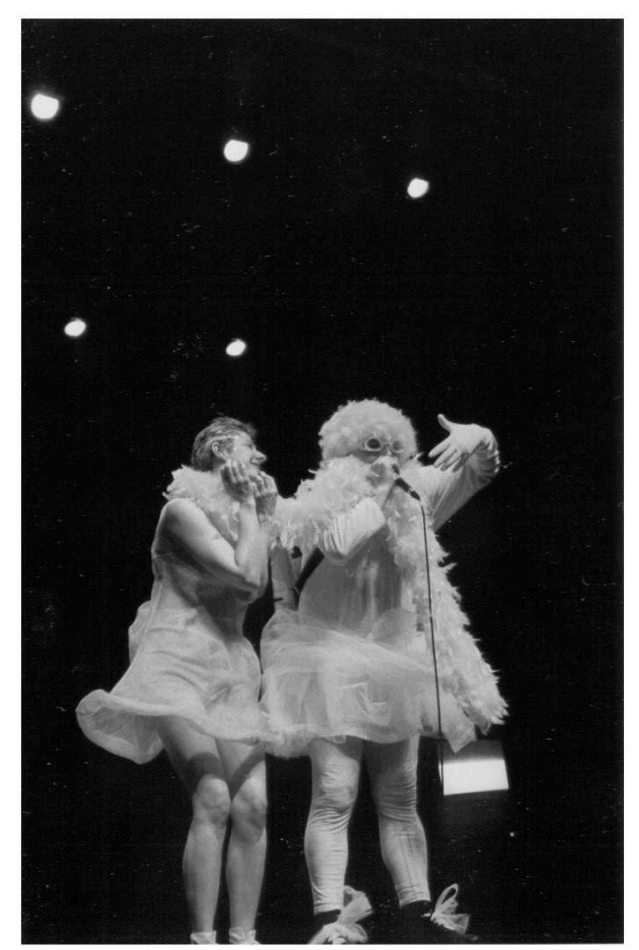

Photo : Joachim Montessuis

\section{Y. R. - LE MOUVEMENT EST-IL, SELON VOUS, UNE EXPRESSION POSITIVE ?}

Le mouvement comme changement de forme, Montagne Froide peut être considérée comme une activité, une activité de variations ${ }^{6}$, de traversée des frontières entre les disciplines. En fait à la suite de Montaigne nous pourrions dire que « nous ne sommes jamais chez nous ». Le mouvement c'est aussi quitter l'idée de centre : décentrement géographique. Au Château des Forges à Pesmes, un bourg dans l'est de la France, nous avons organisé avec l'association du Château des Forges, Les harmoniques, une journée d'étude pour les professionnels et artistes, acteurs dans le domaine de l'édition et créateurs de formes hybrides-décentrement de la pratique - et une soirée de performances et de poésie orale qui ouvrait une exposition consacrée au livre d'artiste - des Avant-Gardes historiques aux expériences récentes. Décentrement en invitant le compositeur Philip Corner et Phoebe Neuville, deux artistes newyorkais, en résidence dans le petit village de Morey, pour construire Sylva Forestis avec Valentine Verhaeghe. Cet événement intermedia fut présenté en forêt et mobilisa perpetuum mobile - les lieux, changeant temporairement quelques formes établies des représentations du quotidien.

\section{Y. R. - PARLEZ-NOUS UN PEU DE VOS ChAMPS ET MOYENS D'EXPRESSION ?}

Comme je vous l'ai dit, nous travaillons à la frontière et au tissage de plusieurs disciplines, à partir de conceptions élargies de l'activité de création, de la respiration au clignement d'œil comme acte de danse, en passant par l'édition, et depuis trois ans l'électronique avec Masahiro Handa, la recherche fondamentale à l'université, l'écriture, ou le jardinage... Arrivons-nous à réaliser des œuvres réellement extramedia ou intermedia? L'habitude des classifications nous aveugle probablement pour 
nous permettre dès à présent d'avancer une réponse. Pour ces œuvres intermédiaires, parfois la notion même d'auteur devient floue, la forme " œuvre » étant une coconstruction, une tension entre plusieurs modules indépendants, une interaction. Nos séjours au Japon nous ont permis de rencontrer il y a plusieurs années d'autres conceptions de l'art, dans la danse particulièrement, et plus largement de l'activité de création, et bien sûr nous avons rencontré des conceptions divergentes de l'idée traditionnelle de l'atelier, ou de la salle de concert dans la fréquentation de courants artistiques occidentaux, tel Fluxus. Finalement la création ne pourrait-elle pas être considérée comme une simple promenade, menée à la manière de cet observateur amusé qui soulignait : «lorsque nous sommes cependant contraints de prendre la même direction à l'aller comme au retour nous remarquons des champignons que nous n'avions pas vus en venant » J. Cage.

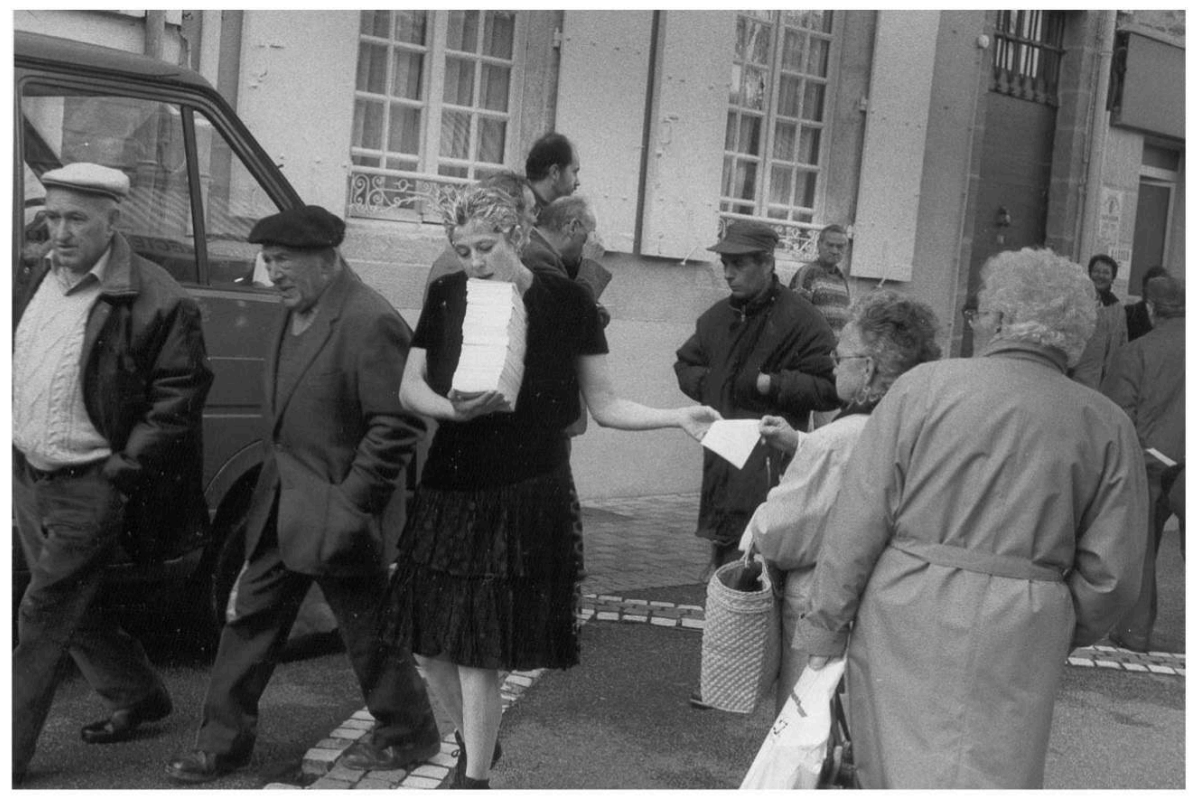

Photo : Corinne Poirier

Quelques rendez-vous avec Montagne Froide :

26 janvier 2002, Misère de l'objet, dans le cadre de l'exposition de J.-B. Farkas, Espace d'art contemporain, Paris $14^{\mathrm{e}}$.

26 février, Impulsion générale, CRAC Le 10 Neuf, Montbéliard.

15 mars, Les attracteurs (Turbulences), performance, suivie d'un débat, par Valentine Verhaeghe, École des Beaux-Arts de Belfort, de 17H00 à $20 \mathrm{H} 15$.

7 avril, Poëm(s), événement autour de l'édition d'artistes, au V.A.C. de Ventabren.

À paraître en avril, Éphéméride, V. Verhaeghe, J. Blaine, M. Messagier, B. Lens, Édition La Main Courante, La Souterraine, publié avec l'aide du Centre national des lettres.

montagne.froide@wanadoo.fr 


\section{NOTES}

1. Écrire millefeuille en pensant aux Mille Plateaux; « hors des strates ou sans les strates, nous n'avons plus ni formes ni substances, ni organisation, ni développement, ni contenu ni expression », DeleuZE, G., GUATTARI, F., Mille Plateaux, Paris, Les Éditions de Minuit, 1980.

2. FILLIOU, R., Théâtre incomplet, Liège, Aarevue de Richard Tialans, 1969-1994, Liège, réédition, Small noise $n^{\circ} 1$, Belgique, 1999.

3. FILLIOU, R., Teaching \& learning as performing arts, Archives Lebeer Hossman, Paris, Bruxelles, 1970, réédition en français, Enseigner et apprendre, arts vivants, Archives Lebeer Hossman, Paris, Bruxelles, 1998.

4. KAPROW, A., L'expérience réelle, in Et tous ils changent le monde, catalogue biennale de Lyon, Lyon, 1993.

5. Le Divers, Séminaire à l'École Nationale des Beaux-Arts de Lyon, 2001/2002.

6. Variations comme variétés, nous retrouvons ici Les Grands Poèmes Faux, variétés, de Matthieu MESSAGIER, Paris, Flammarion, 2000.

\section{AUTEUR}

\section{YOSSI ROSENBOIM}

Membre fondateur du Collectif Artstoria à New York. 